\title{
Wilson loops in large- $N$ theories
}

\author{
Hirosi Ooguri \\ 366 Le Conte Hall, Department of Physics, University of California at Berkeley, Berkeley, \\ CA 94720, USA \\ and \\ Theory Group, Mail Stop 50A-5101, Physics Division, Lawrence Berkeley National Laboratory, \\ Berkeley, CA 94720, USA
}

Received 16 September 1999

Abstract. We discuss how various aspects of Wilson loops in large- $N$ gauge theories are studied from the point of view of the AdS-CFT correspondence.

PACS numbers: $1115 \mathrm{~T}, 1125$

\section{Introduction}

In the AdS-CFT correspondence, correlation functions of conformal fields are related to amplitudes of a quantum theory in anti-de Sitter (AdS) theory [1-3] (for a review, see, for example, [4]). Since the conformal group of the boundary and the isometry group of AdS are identical, correlation functions defined in this way are conformally symmetric. However, not all quantum theories in AdS can be related to conformal field theory (CFT) at the boundary in this way. The correlation functions on the boundary must obey the axioms of CFT. For example, any CFT contains the energy-momentum tensor in the operator algebra, and one must be able to compute correlation functions including the energy-momentum tensor. There has to be a field in AdS to which it can couple, namely the graviton. Thus a quantum theory dual to CFT is necessarily gravitational. A quantum gravity may or may not be a string theory. In some cases, however, there are operators in CFT which can directly couple to string states. Wilson loop operators are examples of such operators. So we may hope to learn about stringy aspects of the AdS-CFT correspondence by studying the Wilson loops. Also, these are fundamental gauge-invariant operators in gauge theories, and we may hope to learn about gauge theories from the point of view of AdS.

In this paper, I will discuss Wilson loops in the $\mathcal{N}=4$ supersymmetric Yang-Mills theory in four dimensions, based on my work with Drukker and Gross [5]. First, we review basic properties of the Wilson loops in $\mathcal{N}=4$ theory. This part of the paper is purely field theoretical. The $\mathcal{N}=4$ theory contains massless scalar fields and they must be taken into account in constructing the Wilson loop. The presence of the scalar fields improves ultraviolet (UV) properties of the loop operators. We will then discuss how these and other properties of Wilson loops can be seen from the point of view of string theory in AdS.

At Strings '99, I also presented my work in progress with Cumrun Vafa about Wilson loops in the large- $N$ Chern-Simons gauge theory in three dimensions, which will appear elsewhere. (This is why the title says 'large- $N$ theories'.) 


\section{Wilson loops in the $\mathcal{N}=4$ super-Yang-Mills theory}

Let me start with the $\mathcal{N}=4$ supersymmetric Yang-Mills theory in four dimensions. As I mentioned, this part of the paper is purely field theoretical.

\subsection{Definition}

The Wilson loop is a phase factor associated with a trajectory of a quark in the fundamental representation of the gauge group $G$. We will discuss the case when $G=U(N)$. The $\mathcal{N}=4$ theory consists of the gauge field $A_{\mu}$, four Weyl fermions $\lambda_{a}(a=1, \ldots, 4)$, and six scalar fields $\phi_{i}(i=1, \ldots, 6)$ all in the adjoint representation of the gauge group $U(N)$. The theory does not contain particles in the fundamental representation. Instead, we may use the W-boson to probe the theory. We start with the $U(N+1)$ gauge group and break it into $U(N) \times U(1)$ by choosing the non-zero vacuum expectation values for the scalar fields,

$$
\phi_{U(N+1)}^{i}=\left(\begin{array}{cc}
\phi_{U(N)}^{i}=0 & 0 \\
0 & u \theta^{i}
\end{array}\right) .
$$

Since there are six scalar fields, we parametrize their vacuum expectation values by a point $\theta^{i}$ on the unit 5 -sphere, $\theta^{2}=1$, corresponding to the direction of the symmetry breaking. The absolute value of the scalar vacuum expectation value is denoted by $u$.

The phase factor associated with a trajectory of the W-boson gives the loop operator of the form,

$$
W=\operatorname{Tr} P \exp \left[\oint \mathrm{d} s\left(\mathrm{i} A_{\mu}(x(s)) \dot{x}^{\mu}(s)+\mathrm{i} \phi_{i}(x(s)) \theta^{i}(s)|\dot{x}(s)|\right)\right]
$$

in the Minkowski space, and

$$
W=\operatorname{Tr} P \exp \left[\oint \mathrm{d} s\left(\mathrm{i} A_{\mu}(x(s)) \dot{x}^{\mu}(s)+\phi_{i}(x(s)) \theta^{i}(s)|\dot{x}(s)|\right)\right],
$$

in the Euclidean space. We should point out that there is an important difference between the Minkowski case and the Euclidean case; that is the absence of the imaginary unit ' $i$ ' in front of the scalar field $\phi$ in the Euclidean case. In particular, the Wilson loop in the Euclidean case is not a pure phase. This distinction is important in many of our results in the following. In this paper, we will deal with the Euclidean case only.

Another important aspect of the Wilson loop (3) generated by the W-boson is that it couples to the gauge field $A_{\mu}$ and the scalar field $\phi_{i}$ with the same strength since $\theta^{2}=1$. Clearly this is a consequence of the fact that the W-boson in this case is a BPS particle. This is not the most general gauge-invariant observable one can write down. In general, one may consider a more general loop operator whose coupling strength to the gauge field may be different from that to the scalar field, as in

$$
W=\operatorname{Tr} P \exp \left[\oint \mathrm{d} s\left(\mathrm{i} A_{\mu}(x(s)) \dot{x}^{\mu}(s)+\phi_{i}(x(s)) \dot{y}^{i}(s)\right)\right] .
$$

Here we use the symbol $\dot{y}^{i}$ to denote the coupling to the scalar field. The phase factor for a $\mathrm{W}$-boson trajectory corresponds to the special case when $\dot{x}^{2}=\dot{y}^{2}$. 


\subsection{UV divergence}

Although the $\mathcal{N}=4$ theory is ultraviolet finite, composite operators of the theory may require regularization. If two local operators coincide at a point, for example, there can be a divergence. Let us discuss the ultraviolet divergence in the Wilson loop operator. It turns out that the operator with the constraint $\dot{x}^{2}=\dot{y}^{2}$ is special in this regard.

If the 't Hooft coupling $g^{2} N$ is small, one may compute the vacuum expectation value of the Wilson loop (4) by the perturbative expansion. The one-loop computation gives

$$
\langle W\rangle=1+\frac{g^{2} N}{(2 \pi)^{2} \epsilon} \oint \mathrm{d} s|\dot{x}|\left(1-\frac{\dot{y}^{2}}{\dot{x}^{2}}\right)+\cdots .
$$

Here $\epsilon$ is the UV cut-off parameter. When $\dot{x}^{2}=\dot{y}^{2}$, the divergence is cancelled due to the cancellation between the gauge field exchange and the scalar field exchange diagrams. It seems that this cancellation persists at higher loops. As we will see in the following, the AdS-CFT correspondence shows that the cancellation of the UV divergence also happens at large $g^{2} N$.

So far, we have assumed that the loop is smooth. When there is a singularity on the loop, the divergence is not completely cancelled, and some logarithmic divergence remains even when $\dot{x}^{2}=\dot{y}^{2}$. For example, when the loop has a cusp, the one-loop computation shows the logarithmic divergence depending on the angle at the cusp as

$$
\left\langle W_{\text {with cusp }}\right\rangle=1+\frac{g^{2} N}{(2 \pi)^{2}} \frac{\pi-\Omega}{\sin \Omega}(\cos \Omega+\cos \Theta) \log \left(\frac{1}{\epsilon}\right)+\cdots,
$$

where $\Omega$ is the angle at the cusp (i.e. a jump in $\dot{x}^{\mu} /|\dot{x}|$ ). At the cusp, the direction $\theta^{i}=\dot{y}^{i} /|\dot{y}|$ of $\dot{y}^{i}$ may also change discontinuously; the angle $\Theta$ is the amount of the discontinuity in $\theta^{i}$ at the cusp. Renormalizing this divergence would then give an anomalous scaling property of the loop, which depends on $\Omega$ and $\Theta$. There is also a logarithmic divergence when the loop has an intersection,

$$
\left\langle W_{\text {with intersection }}\right\rangle=1+\frac{g^{2} N}{2 \pi} \frac{1}{\sin \Omega}(\cos \Omega+\cos \Theta) \log \left(\frac{1}{\epsilon}\right)+\cdots .
$$

These are somewhat similar to the logarithmic divergence in the even-dimensional observables (such as points or surfaces) discussed by Berenstein et al [6] and by Graham and Witten [7].

\subsection{Loop equation}

The large- $N$ loop equation is considered to be one of the fundamental properties of the Wilson loop operators [8]. It is expected to hold in both small and large $g^{2} N$. When $g^{2} N$ is small, it is equivalent to the perturbative Feynmann rules of the gauge theory. On the other hand, there are subtleties in the definition of the loop equation since it is derived assuming that the loops are regularized but not renormalized. In fact, the equation depends explicitly on the UV cut-off. The situation seems better in the $\mathcal{N}=4$ theory because of the better ultraviolet behaviour, as we saw in the above. Since the equation is also supposed to hold at large $g^{2} N$, it may be useful in testing the stringy aspects of the AdS-CFT correspondence. I will comment on this later in this paper.

In order to write down the loop equation, we need a complete set of gauge-invariant observables which can be written in the form of loops. In particular, we also need to introduce sources for the fermion fields. From the point of view of supersymmetry, it is natural to couple the fermionic variable $\zeta$ to the gluino field $\lambda$ in the combination,

$$
W=\operatorname{Tr} P \exp \left[\oint \mathrm{d} s\left(\mathrm{i} A_{\mu} \dot{x}^{\mu}+\phi_{i} \dot{y}^{i}+\frac{1}{2} \mathrm{i} \bar{\zeta}\left(\mathrm{i} \gamma_{\mu} \dot{x}^{\mu}+\Gamma_{i} \dot{y}^{i}\right) \lambda+\cdots\right)\right] .
$$


It turns out that the combination $\left(\mathrm{i} \gamma_{\mu} \dot{x}^{\mu}+\Gamma_{i} \dot{y}^{i}\right)$ of $4 d$ and $6 d$ gamma-matrices, $\gamma_{\mu}$ and $\Gamma_{i}$, becomes nilpotent when the constraint $\dot{x}^{2}=\dot{y}^{2}$ is satisfied. This simplifies our task of writing down the loop equation considerably. We then introduce the second-order differential operator on the loop space defined as $\dagger$

$\mathcal{L}=\lim _{\eta \rightarrow 0} \oint \mathrm{d} s \int_{s-\eta}^{s+\eta} \mathrm{d} s^{\prime}\left(\frac{\delta^{2}}{\delta x^{\mu}(s) \delta x^{\mu}\left(s^{\prime}\right)}-\frac{\delta^{2}}{\delta y^{i}(s) \delta y^{i}\left(s^{\prime}\right)}+\frac{\delta^{2}}{\delta \zeta(s) \delta \bar{\zeta}\left(s^{\prime}\right)}\right)$.

The two derivatives are taken at different points $s$ and $s^{\prime}$ on the loop. The distance $\eta$ between the two points must be chosen to be shorter than the UV cut-off $\epsilon$ so that we can isolate the contact terms we need for the loop equation. This is the prescription due to Polyakov [9].

When the loop satisfies the constraint $\dot{x}^{2}=\dot{y}^{2}$, the action of the differential operator on the Wilson loop can be written, using the Schwinger-Dyson equation and the large- $N$ factorization, as

$\mathcal{L}\langle W\rangle=g^{2} N \oint \mathrm{d} s \oint \mathrm{d} s^{\prime} \delta^{(4)}\left(x(s)-x\left(s^{\prime}\right)\right)\left[\dot{x}(s) \cdot \dot{x}\left(s^{\prime}\right)-\dot{y}(s) \cdot \dot{y}\left(s^{\prime}\right)\right]\left\langle W_{s s^{\prime}}\right\rangle\left\langle W_{s^{\prime} s}\right\rangle$,

where $W_{s s^{\prime}}$ is given by the path-ordered exponential of the form (8) integrated over the part of the loop, between $s$ and $s^{\prime}$. The fermionic variables $\zeta$ are set to be zero after taking the derivative. The loop equation (10) states that $\mathcal{L}\langle W\rangle$ is non-zero only when the loop has a self-intersection. In the case of pure Yang-Mills theory without supersymmetry, there is an ambiguity about whether to take into account the trivial self-intersection, namely the case when $s=s^{\prime}$, for which the delta-function constraint $x^{\mu}(s)=x^{\mu}\left(s^{\prime}\right)$ in the right-hand side of (10) is satisfied trivially. In some sense, the loop intersects with itself at each point along the loop. In the case of the $\mathcal{N}=4$ theory, we do not have to worry about such an ambiguity since the factor $\left[\dot{x}(s) \cdot \dot{x}\left(s^{\prime}\right)-\dot{y}(s) \cdot \dot{y}\left(s^{\prime}\right)\right]$ vanishes when $s=s^{\prime}$.

\section{Wilson loops in $A d S_{5} \times S^{5}$}

Now let us discuss how these properties of loop operators can be seen from the point of view of a string in $\mathrm{AdS}$. In the above, we started with the $U(N+1)$ gauge group and broke the group into $U(N) \times U(1)$. In string theory, this corresponds to putting $N$ D3-branes on top of each other, and probing it with another D3-brane. The open string stretched between the $N$ D3-branes and the single D3-brane probe corresponds to the W-boson of the gauge theory. According to Maldacena's conjecture, in the large- $N$ limit, the $N$ D3-branes are replaced by the geometry of the $A d S_{5}$ times a 5-sphere. The W-boson is now a string in AdS stretched from the boundary. The large- $N$ Wilson loop was studied from this point of view by Maldacena [11] and by Rey and Yee [12]. In the following we will clarify some aspects of this approach and extend it to various cases.

The metric on $A d S_{5}$ times the 5-sphere is given by

$$
\mathrm{d} s^{2}=\sqrt{g^{2} N} y^{-2}\left(\mathrm{~d} y \mathrm{~d} y+\mathrm{d} x^{\mu} \mathrm{d} x^{\mu}\right)+\sqrt{g^{2} N} \mathrm{~d} \theta^{2} .
$$

It is often useful to combine the radial coordinate $y$ of AdS with the coordinates $\theta$ of the 5 -sphere into six coordinates $y^{i}=y \theta^{i}$. In the coordinates $x^{\mu}$ and $y^{i}$, it is easy to see that the total metric is conformal to the flat ten-dimensional metric,

$$
\mathrm{d} s^{2}=\sqrt{g^{2} N} y^{-2}\left(\mathrm{~d} x^{\mu} \mathrm{d} x^{\mu}+\mathrm{d} y^{i} \mathrm{~d} y^{i}\right) .
$$

In these coordinates, the boundary of $\mathrm{AdS}$ is at $y=0$.

$\dagger$ The differential operator $\mathcal{L}$ defined here does not preserve the constraint $\dot{x}^{2}=\dot{y}^{2}$. Recently some improvement in the definition of $\mathcal{L}$ was made, and it was found to be possible to write a loop equation which closes only among loops preserving the constraint [10]. 


\subsection{Boundary conditions}

To compute the Wilson loop observables in this framework, it is important to understand the relation between the loop variables and the boundary condition on the string worldsheet. It is well known that the conformal field theory on the string worldsheet couples to the spacetime gauge fields $A_{\mu}$ and $\phi_{i}$ as

$$
\oint \mathrm{d} \sigma^{1}\left(A_{\mu} \frac{\partial X^{\mu}}{\partial \sigma^{1}}+\phi_{i} P^{i}\right)
$$

where the integral is along the boundary of the worldsheet. We use $\sigma^{\alpha}(\alpha=1,2)$ for the worldsheet coordinates with the worldsheet boundary at $\sigma^{2}=0$. The vector field $A_{\mu}$ couples to the derivative of the string coordinates $X^{\mu}$ along the boundary of the worldsheet. The scalar fields $\phi_{i}$, on the other hand, couple to the momentum $P^{i}$ conjugate to the corresponding string coordinates $Y^{i}$,

$$
P^{i}=\frac{1}{\sqrt{g}} g_{1 \alpha} \epsilon^{\alpha \beta} \frac{\partial Y^{i}}{\partial \sigma^{\beta}},
$$

where $g_{\alpha \beta}$ is a metric on the worldsheet. The scalar fields $\phi_{i}$ correspond to the transverse coordinates of the D-brane, and they are T-dual of the gauge field along the D-brane. Since the momentum $P^{i}$ is T-dual to the derivative of the string coordinate along the boundary, the scalar fields couple to $P^{i}$.

Since the gauge field $A_{\mu}$ couples to the derivative of the string coordinates $\partial_{1} X^{\mu}$ along the boundary and the scalar field $\phi_{i}$ couples to the momentum $P^{i}$ conjugate to the string coordinates at the boundary, it is clear that the Wilson loop operator of the form (4) couples to the string worldsheet with the following boundary conditions:

$$
\begin{aligned}
& X^{\mu}\left(\sigma^{1}, \sigma^{2}=0\right)=x^{\mu}\left(\sigma^{1}\right) \\
& P^{i}\left(\sigma^{1}, \sigma^{2}=0\right)=\frac{\mathrm{d} y^{i}}{\mathrm{~d} \sigma^{1}}\left(\sigma^{1}\right) .
\end{aligned}
$$

In the four-dimensional directions along the D3-brane, the string worldsheet obeys the Dirichlet condition that the string ends along the loop $x^{\mu}(s)$. For the six transverse directions, the string momentum is fixed following the Neumann condition. These boundary conditions are complementary to the standard D3-brane boundary conditions (the Neumann condition for $X^{\mu}$ and the Dirichlet condition for $Y^{i}$ ), as they should be since we are imposing extra conditions by inserting the loop operator on the D-brane.

When $g^{2} N$ is large, the string tension becomes large and we can approximate the string dynamics by a minimum surface in $\operatorname{AdS}[11,12]$. For a given set of the loop variables $\left(x^{\mu}(s), y^{i}(s)\right)$, we expect that there is a unique minimum surface in AdS obeying these boundary conditions. The existence and uniqueness of minimum surfaces in AdS, in the case when $\dot{y}^{i}$ is constant, have been discussed in the mathematics literature (see, for example, [13-15]). This, however, leads to a puzzle. In the AdS-CFT correspondence, we expect that the boundary conditions for the bulk degrees of freedom are imposed at the boundary of AdS at $y=0$. However, the condition for the string worldsheet to terminate at $y=0$ would be an extra Dirichlet boundary condition. This may or may not be compatible with the ten Dirichlet/Neumann boundary conditions we have already imposed.

It turns out that there is a nice resolution to this puzzle. One can show that, if the boundary conditions are smooth, the minimum surface obeying these ten boundary conditions can terminate at $y=0$ only if the constraint $\dot{x}^{2}=\dot{y}^{2}$ is satisfied [5]. This can be shown by using the Hamilton-Jacobi equation for a minimum surface in AdS. This fits nicely with the 
fact we saw earlier in the field theory point of view; the Wilson loop that is generated by a trajectory the W-boson obeys the same constraint $\dot{x}^{2}=\dot{y}^{2}$. This resolves the puzzle but it raises another question about how to define the Wilson loop operator which does not obey the constraint. We will return to this question later.

\subsection{Legendre transformation}

Once we find the minimum surface obeying the boundary condition, we can compute the value of its classical action. In the semiclassical approximation, the vacuum expectation value of the Wilson loop is given by the exponential of the action for the minimum surface. There is a question of which action to use. A naive guess would be the area of the surface, namely the Nambu-Goto action. This would be appropriate if we were solving the fully Dirichlet problem. In the fully Dirichlet problem, the boundary loop is fixed in the target space, and there is a well defined area for each surface. The area, however, is not an appropriate action functional for the Neumann problem. Since the Neumann problem fixes the string momentum, rather than the location of the loop at the boundary, the area for the surface is not well defined. The appropriate action for the Neumann problem is the Legendre transform of the area, which we denote by $\tilde{A}$.

$$
\tilde{A}=A-\oint \mathrm{d} s P_{i} Y^{i}
$$

$\tilde{A}$ obtained by the Legendre transformation is a good functional of the string momentum.

There is a bonus in performing this Legendre transformation. Since the metric in AdS diverges near the boundary, the area of the minimum surface is infinite if the surface terminates at the boundary of AdS. To regularize this, we introduce a cut-off $\epsilon$ in the $y$-coordinates. The boundary of AdS is at $y=0$, and the regularized area is given by an integral in the region $y \geqslant \epsilon$. By now, it is well known that this infrared regularization in AdS corresponds to the ultraviolet regularization in the gauge theory [16]. So we use the same symbol $\epsilon$ for both the UV cut-off of the gauge theory and the IR cut-off of the string theory in AdS. If the loop is smooth, the area is linearly divergent and the divergence is proportional to the circumference of the loop [11].

$$
A=\frac{\sqrt{g^{2} N}}{\epsilon} \oint \mathrm{d} s|\dot{x}|+(\text { finite }) .
$$

It turns out that the Legendre transformation precisely cancels this linear divergence, leaving the Legendre transformed action $\tilde{A}$ to be finite. Therefore, the vacuum expectation value of the Wilson loop, which is given by the exponential of $\tilde{A}$, is finite in this case. Earlier, we have seen in the gauge theory that the Wilson loop is perturbatively finite when $\dot{x}^{2}=\dot{y}^{2}$. The fact that $\tilde{A}$ is finite fits well with this gauge theory result.

\subsection{Examples}

There are several types of Wilson loops for which solutions to the corresponding minimum surface problems can be found explicitly and the areas of the surfaces can be computed analytically.

The first example is the parallel Wilson lines. This was studied by Maldacena [11] and by Rey and Yee [12]. By computing the area of the minimum surface connecting the Wilson lines and by performing the Legendre transformation, one finds

$$
\tilde{A}=\sqrt{g^{2} N} \frac{4 \pi \sqrt{2}}{\Gamma\left(\frac{1}{4}\right)^{4}} \frac{L}{R},
$$


where $L$ is the length of the Wilson lines and $R$ is the distance between them. This expression is for $L \gg R$. The parallel Wilson lines compute the potential between the quark and antiquark. This result shows that the potential goes as $1 / R$, as expected from the conformal invariance, and the coefficient is proportional to $\sqrt{g^{2} N}$. It is interesting to compare this with the perturbative computation. When $g^{2} N$ is small, the quark-antiquark potential is proportional to $g^{2} N$ due to that gluon exchange. Somehow when $g^{2} N$ becomes large, this $g^{2} N$ behaviour turns into $\sqrt{g^{2} N}$. One may view this as a prediction of the AdS-CFT correspondence, which can in principle be tested by a field theory computation at large $N$.

We can also find a minimum surface corresponding to a circular Wilson loop. The area of the surface, after performing the Legendre transformation, turns out to be independent of the radius of the circle, and the vacuum expectation value of the loop is given by

$$
\langle W\rangle=\exp \left(\sqrt{g^{2} N}\right) .
$$

In these two cases, the Wilson loops are finite as we expect for smooth loops.

Another case for which we can find a minimum surface is a loop with a cusp. Near the cusp singularity, the geometry is scale invariant and we can integrate the equation of motion using the elliptic integrals. In this case, the divergence of the area is not precisely cancelled by the Legendre transformation, and the logarithmic divergence remains. Once again, this is similar to what we saw in the gauge theory side (6). The coefficient in front of the logarithm is different from the perturbative result, however.

\subsection{Loops with $\dot{x}^{2} \neq \dot{y}^{2}$}

We have seen that, when the Wilson loop operator obeys the constraint $\dot{x}^{2}=\dot{y}^{2}$, we can evaluate its vacuum expectation value at large $g^{2} N$ by computing the area of the minimum surface in AdS. Its vacuum expectation value is the exponential of the Legendre transform of the area, and it is finite when the loop is smooth.

It is then natural to ask how to compute the loop which does not satisfy the constraint. For the boundary conditions which do not satisfy $\dot{x}^{2}=\dot{y}^{2}$, there is no minimum surface ending on the boundary of AdS. So one may say that the vacuum expectation value of such a Wilson loop should be zero. This is a reasonable answer. In fact, in other cases such as finite-temperature theories, such an answer gave results consistent with what we expect for gauge theories [17].

For some problems, however, we need more detailed information than simply stating $\langle W\rangle=0$ for $\dot{x}^{2} \neq \dot{y}^{2}$ at large $g^{2} N$. Suppose, for example, we want to see whether the Wilson loop computed in this way gives a solution to the large- $N$ loop equation (10). When the loop is smooth and without intersections, the equation is simply $\mathcal{L}\langle W\rangle=0$ and this is satisfied by any smooth functional of the loop. Non-trivial checks of the loop equation, therefore, have to involve loops with cusps or intersections. For a loop with a cusp, however, a minimum surface which can end at the boundary of AdS violates the condition $\dot{x}^{2}=\dot{y}^{2}$ [5], while the loop equation (10) is derived for loops obeying $\dot{x}^{2}=\dot{y}^{2}$. Thus, in order to test the loop equation, we need more refined knowledge on the vacuum expectation value of such Wilson loops.

The perturbative computation suggests that loops not obeying the constraint are ultraviolet divergent. In analogy with the distinction between chiral primary fields and non-chiral fields in gauge theory, we expect that computation of the vacuum expectation value for loops with $\dot{x}^{2} \neq \dot{y}^{2}$ requires a better understanding of stringy corrections in AdS. 


\section{Comments}

At the end of my presentation at Strings '99, I was asked whether the Wilson loop operator $W$ as in (4) is well defined in the Euclidean quantum field theory. Do we know that the functional integral for $\langle W\rangle$ is convergent? Since the scalar field $\phi_{i}$ in the exponent comes with the real coefficient $\dot{y}^{i}$, the functional integral would be convergent only if the distribution of the eigenvalues of $\phi_{i}$ decays sufficiently quickly for large eigenvalues. Another audience commented that, since the Wilson loop with the constraint $\dot{x}^{2}=\dot{y}^{2}$ is BPS-like (it is a phase factor associated with a trajectory of the W-boson, which is a BPS particle in the $\mathcal{N}=4$ theory), it is likely that such an operator makes sense, and so does that with $\dot{x}^{2}>\dot{y}^{2}$ since the effect of $\phi_{i}$ in the exponent would be weaker. On the other hand, one may question whether an operator with $\dot{x}^{2}<\dot{y}^{2}$ exists.

In fact, the AdS-CFT correspondence suggests that operators with $\dot{x}^{2}=\dot{y}^{2}, \dot{x}^{2}>\dot{y}^{2}$ and $\dot{x}^{2}<\dot{y}^{2}$ behave differently. As I pointed out, the minimum surface can terminate at the boundary of AdS at $y=0$ only if the constraint $\dot{x}^{2}=\dot{y}^{2}$ is satisfied. The AdSCFT correspondence then gives a definite prescription to compute $\langle W\rangle$ using the Legendre transform of the area of the minimum surface. When $\dot{x}^{2}>\dot{y}^{2}$, we can still find a minimum surface obeying the boundary conditions (15), except that the surface ends somewhere in the interior of AdS rather than at the boundary. One may therefore hope to compute $\langle W\rangle$ using such a minimum surface. On the other hand, in the case of $\dot{x}^{2}<\dot{y}^{2}$, there is no solution to the minimum surface problem even if we relax the condition that the surface should terminate at $y=0$. This may be viewed as an indication that the loop operator for $\dot{x}^{2}<\dot{y}^{2}$ is problematic. It would be interesting to study properties of such loops from the point of view of the gauge theory and to see how they fit with these behaviours of minimum surfaces.

To conclude, the Wilson loop provides us with a window for observing the stringy nature of the correspondence between gauge theory and string theory. In the $\mathcal{N}=4$ gauge theory in four dimensions, we have understood various aspects of loops which obey the constraint, $\dot{x}^{2}=\dot{y}^{2}$. I think that finding a way to study loops without the constraint would teach us more about gauge theory and string theory.

\section{Acknowledgments}

It is my pleasure to thank the organizers of Strings ' 99 for giving me the opportunity to present this work at the conference and for their hospitality. I would like to thank Nadav Drukker and David Gross for their collaboration on this work. This research is supported in part by NSF grant PHY-95-14797 and DOE grant DE-AC03-76SF00098.

\section{References}

[1] Maldacena J 1998 Adv. Theor. Math. Phys. 2231 (Maldacena J 1997 Preprint hep-th/9711200)

[2] Gubser S S, Klebanov I R and Polyakov A M 1998 Phys. Lett. B 428105 (Gubser S S, Klebanov I R and Polyakov A M 1998 Preprint hep-th/9802109)

[3] Witten E 1998 Adv. Theor. Math. Phys. 2253 (Witten E 1998 Preprint hep-th/9802150)

[4] Aharony O, Gubser S S, Maldacena J, Ooguri H and Oz Y 2000 Phys. Rep. 323183 (Aharony O, Gubser S S, Maldacena J, Ooguri H and Oz Y 1999 Preprint hep-th/9905111)

[5] Drukker N, Gross D J and Ooguri H 1999 Phys. Rev. D 60125006 (Drukker N, Gross D J and Ooguri H 1999 Preprint hep-th/9904191)

[6] Berenstein D, Corrado R, Fischler W and Maldacena J 1999 Phys. Rev. D 59105023 (Berenstein D, Corrado R, Fischler W and Maldacena J 1998 Preprint hep-th/9809188) 
[7] Graham C R and Witten E 1999 Nucl. Phys. B 54652

(Graham C R and Witten E 1999 Preprint hep-th/9901021)

[8] Migdal A A 1983 Phys. Rep. 102199

[9] Polyakov A M 1987 Gauge Fields and Strings (Geneva: Harwood) section 7.2

[10] Drukker N 1999 J. High Energy Phys. JHEP11(1999)006

(Drukker N 1999 Preprint hep-th/9908113)

[11] Maldacena J 1998 Phys. Rev. Lett. 804859

(Maldacena J 1998 Preprint hep-th/9803002)

[12] Rey S-J and Yee J 1998 Preprint hep-th/9803001

[13] Anderson M 1982 Invent. Math. 69477

[14] Lin F-H 1989 Invent. Math. 96593

[15] Tonegawa Y 1996 Math. Z. 221591

[16] Susskind L and Witten E 1998 Preprint hep-th/9805114

[17] Aharony O and Witten E 1998 J. High Energy Phys. JHEP11(1998)018

(Aharony O and Witten E 1998 Preprint hep-th/9807205) 